Z. Klin. Chem. Klin. Biochem.

11. Jg. 1973, S. 398-402

\title{
Die Chymosinaktivität der Proteasen des Magen- und Duodenal-Saftes nach der elektrophoretischen Fraktionierung im Agar-Gel
}

\author{
Von J. Kamarít und Véra Mechloví \\ Aus dem Forscbungsinstitut für Pädiatrie (Direktor: Doz. MUDr. O. Billek, C. Sc.) Brno (CSSR)
}

(Eingegangen am 4. Dezember 1972/21. April 1973)

Es wird ein „Sandwich“-Detektionsverfahren der Chymosinaktivitäten der Magen- und Duodenal-Saft-Proteasen nach der Agar-GelElektrophorese beschrieben. Mit dem Substrat Casein ist es möglich, auf Grund der Chymosin-Wirkung der Magen- und PankreasProteasen alle diese Enzyme mit einem einzigen Substrat und bei gleichen pH-Bedingungen nebeneinander auf einem Zymogramme nachzuweisen.

The chymosin activity of the gastric and duodenal juice proteinases separated by means of agar gel electrophoresis

A "sandwich" detection technique for the chymosin activity of gastric and duodenal juice proteases separated by means of agar gel electrophoresis is described. On the basis of their chymosin activity it is possible to detect all these enzymes in the reaction with the substrate casein in one zymogram under the same $\mathrm{pH}$ condition.

Im Verlauf der letzten zehn Jahre haben verschiedene Autoren den heterogenen Charakter der Magen- sowie auch Pankreas-Proteasen gezeigt. Eine glänzende ausführliche Übersicht über die Heterogenität des Pepsinogens und Pepsins hat SAmLoff (1) veröffentlicht. Zwei Studien über einige Formen des Chymotrypsins haben COAN et al. publiziert $(2,3)$. Was das Trypsin betrifft, haben Robinson et al. (4) im Pankreas-Saft zwei aus zwei verschiedenen Zymogenen stammende Trypsine gefunden, während von CoAN et al. (2) nur ein Trypsin nachgewiesen wurde.

Vor einiger Zeit haben wir eine Methode für die Fraktionierung der Pepsinogene und Pepsine des Magens mit der Agar-Gel-Elektrophorese und der sogenannten „Sandwich"-Detektionstechnik veröffentlicht (5). Die zuletzt eingeführte Technik ermöglicht den Nachweis der Chymosin-Aktivitäten, welche die Fraktionen der einzelnen Proteasen ebenso aufweisen. In der vorliegenden Arbeit werden die auf Grund der proteolytischen Aktivität entwickelten Zymogramme des Pepsinogens und des Pepsins mit den parallelen Zymogrammen mit Chymosin-Aktivitäten der erwähnten Enzyme verglichen. Die Protease-Fraktionen des Duodenums, die Chymosin-Aktivität aufweisen, werden den mit spezifischen synthetischen Substraten entwickelten Aktivitäten für Trypsin und Chymotrypsin gegenübergestellt.

\section{Material und Methoden}

Die Enzym-Aktivitäten wurden in den übliçherweise entnommenen Magen- und Duodenal-Säften der Patienten, bei welchen die Sondierung auf Grund verschiedener gastrointestinaler Störungen indiziert war, verfolgt. Bei der Duodenalsaft-Entnahme wurden Magnesiumsulfat und Vitamin A als Stimulantia benutzt. Beide Stimulantia wurden in den bei einigen Fällen ausführlich angeführten Mengen (siehe Text der Abbildungen) durch die Sonde verabfolgt. Die Magenschleimhaut-Proben wurden aus dem menschlichen Sektions-Material nicht später als 24 Stunden nach dem Tode gewonnen ${ }^{1}$ ). Als Kontrolle haben wir auch die proteolytischen Aktivitäten der Pepsinogen- und Pepsin-Fraktionen in dem mit bioptischer Sonde entnommenen Material verfolgt und haben im Vergleich mit relativ frischem nekroptischem Material entsprechende Ergebnisse bekommen. Für die elektrophoretische Fraktionierung wurden hier die Magenschleimhaut-Extrakte im Phosphat-Puffer $\mathrm{pH} 6$ benutzt.

Die Technik der elektrophoretischen Pepsinogen- und PepsinFraktionierung haben wir früher ausführlich beschrieben (5). In gleicher Weise wird auch die Elektrophorese der DuodenalsaftEnzyme durchgeführt.

Noch während der elektrophoretischen Fraktionierung werden die Agar-Gel-Detektionsschichten vorbereitet. Diese bestehen aus dem Agar $(10 \mathrm{~g} / 1)$ in $2 \mathrm{mmol} / \mathrm{l}$ Phosphat-Puffer pH $6 \mathrm{mit} 2 \mathrm{mmol} / \mathrm{l}$ Endkonzentration an $\mathrm{CaCl}_{2}$ und 17,5 g/l der aufgelösten fettfreien Trockenmilch ${ }^{2}$ ). Nach der Fraktionierung werden die Elektrophorese-Agar-Gel-Plättchen für $15 \mathrm{~min}$ in Phosphat-Puffer (Elektrophoresepuffer) eingetaucht. Während dieser Zeit sollen die die Detektion störenden, Artefakte-bildenden Stoffe aus der Elektrophoreseschicht ausgeschwemmt werden. Diẹ überflüssige Pufferlösung wird mit Filterpapier abgetupft. Auf die so vorbereiteten Agar-Gel-Schichten werden die Detektionsschichten gelegt. Die so entstandenen "Sandwichs" werden einem feuchten Milieu bei $37^{\circ} \mathrm{C}$ so lange ausgesetzt $(60-120 \mathrm{~min})$, bis die Fraktionen mit Chymosin-Aktivitäten als dichtes Präzipitat erscheinen. Im Falle des Trypsins und Chymotrypsins hellt sich dieses Präzipitat durch die folgende proteolytische Aktivität früher oder später auf. Für die Identifizierung des Trypsins und Chymotrypsins des Duodenal-Saftes wird das ursprüngliche Enzymelektropherogramm quer in $5 \mathrm{~mm}$ breite Streifen geschnitten. Die Streifen werden in Inkubationsmilieu eingetaucht und eluiert. Die Zusammensetzung dex Inkubationslösung zur Chymotrypsindetektion: $3 \mathrm{ml} 0,5 \mathrm{~mol} / \mathrm{l}$ Tris-Puffer $\mathrm{pH} 7,5 ; 66 \mathrm{mmol} / \mathrm{l} \mathrm{CaCl} \mathrm{Can}_{2}$ und $0,1 \mathrm{ml}$ $0,13 \mathrm{~mol} / 1$ Carboxypropionyl-phenylalanin- $p$-nitranilid. Inkubationslösung zur Trypsindetektion: $2,5 \mathrm{ml} 0,2 \mathrm{~mol} / 1$ Tris-Puffer, $\mathrm{pH} 7,8 ; 25 \mathrm{mmol} / 1 \mathrm{CaCl}_{2}$, dazu wird $0,3 \mathrm{ml} 10 \mathrm{mmol} / 1$ Benzoylarginin- $p$-nitranilid zugefügt. Beide Substrate wurden von der Firma BOEHRINGER bezogen. Die verschlossenen Reagenzgläser werden

1) Für das überlassene Material danken wjr Herrn Dr. B. HabaNEC, II. Institut für Pathologische Anatomie des Universitätskinderkrankenhauses Brno.

2) Präparat LAKTINO, Industrie für Milchernährung, CSSR. 
16 Stunden im Thermostat bei $37^{\circ} \mathrm{C}$ inkubiert. Anschließend wird dic Extinktion der Lösung in der Küvette bei $405 \mathrm{~nm}$ gegen Leerwert gemessen. Als Leerwert dient die Inkubationslösung, in welcher ein $5 \mathrm{~mm}$ breiter Agar-Gel-Streifen ohne Enzym-Aḳtivität cluiert wurde.

\section{Ergebnisse und Diskussion}

Nach der Agar-Gel-Elektrophorese weist das Pepsin des Magensaftes zwei Fraktionen auf. Die Fraktion näher der Anode erscheint stets mit zweimal so hoher Aktivität wie die zweite, langsamer wandernde Fraktion. Diese Fraktion verliert im $\mathrm{pH}$ Bereich 6,5 ihre Aktivität, die schneller anodisch wandernde Fraktion verliert dagegen die Aktivität bis zum pH Bereich 7. In seltenen Fällen haben wir auch die umgekehrte Situation in dem kritischen pH-Bereich 6,5-7,0 beobachtet (Abb. 1). In diesem Zusammenhang verweisen wir auf die Arbeit von SEIJFFERS et al., in welcher die Autoren mittels $\mathrm{pH}$ 7,1 das Magen-Pepsin in alkali-stabil und alkali-labil differenziert haben $(6,7)$. Aus den oben erwähnten Angaben geht deutlich hervor, daß im normalen alkalisch reagierenden Duodenalsaft keine Pepsin-Aktivitäten mehr nachweisbar sind. Was die Chymosin-Aktivität des Pepsins betrifft, decken sich die Fraktionen mit den Protease-Aktivitäten des Pepsins, welche im stark sauren Milieu beobachtet wurden. Die Chymosin-Aktivität weist aber eine größere Resistenz im $\mathrm{pH}$ Bereich 6,5-7,0 auf, so daß unter Bedingungen, in welchen die eine oder beide proteolytische Aktivitäten des Pepsins nicht mehr sichtbar sind, die Chymosinaktivitäten noch nachweisbar sind (Abb. 2). Demzufolge können in neutral reagieren-

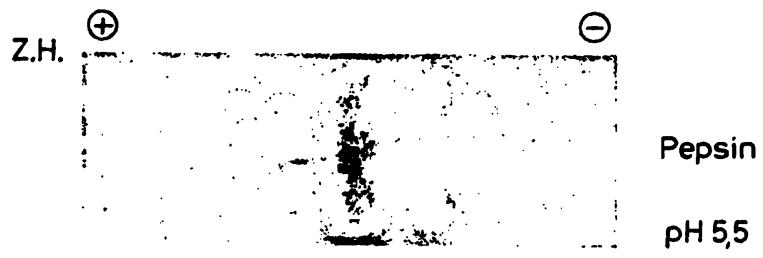

$\mathrm{pH} 6,5-6,8$

K.M. .

Pepsin

$\mathrm{pH} 6,0$

$\mathrm{pH} 6,5-6,8$

Start

Abb. 1

Die Aktivitäten der Pepsin-Isoenzyme des Magensaftes im kritischen pH-Bereich 6,5-6,8. Siehe Text
$\mathrm{KL}$.

a

$\mathrm{pH} 2$

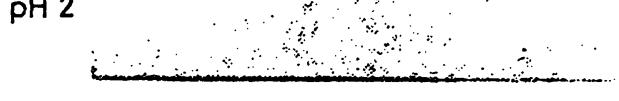

$\oplus$

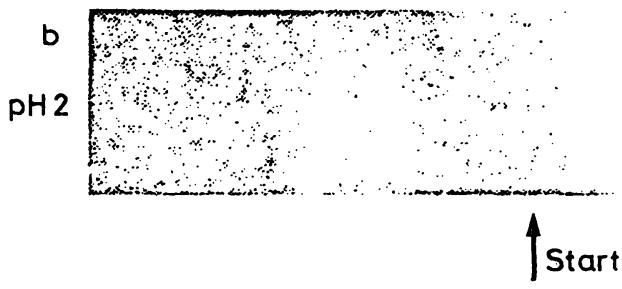

KO.
KU.

$\oplus$

$\Theta$

$\mathrm{pH} 4,5$

a

$\oplus \quad \Theta$

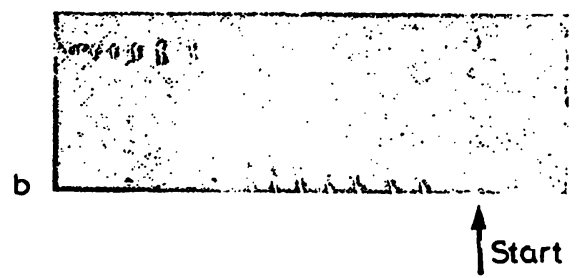

Abb. 2

pH 6,5 Die proteolytischen (a) und Chymosin-Aktivitäten (b) des Magensaftes von drei Kranken. Die angegebenen pH-Werte sind die ursprünglichen Wer
menen Magensäfte 


\section{K.Z.}
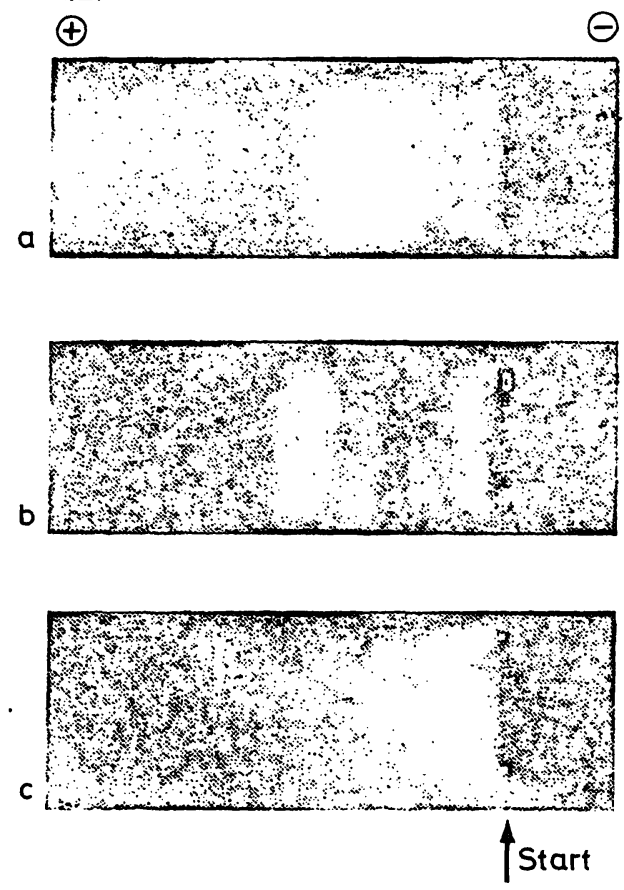

Abb. 3

Die Chymosin-Aktivitäten der Proteasen in drei Proben des Duodenalsaftes von einem Patienten. a: ohne Stimulation; b: nach MgSO $(15 \mathrm{ml}, 250 \mathrm{~g} / \mathrm{l})$; c: nach Vitamin A Stimulation (10000 $\mathrm{I}$. E.). In a und $b$ entsprechen die zwei am schnellsten wandernden Fraktionen den Chymosin-Aktivitäten der Pepsin-Isoenzyme

den oder schwach alkalischen Duodenalsäften mit Hilfe der Chymosin-Aktivitäts-Detektion mit dem Substrat Casein die Chymosin-Aktivität der Fraktionen des Magenpepsins als. auch die Chymosin-Aktivität der Pankreas-Proteasen nebeneinander identifiziert wer- den. Die auf Grund der Chymosin-Wirkung der Proteasen entstandenen Fraktionen des Paracaseins werden im Laufe längerer Inkubationszeit durch die im $\mathrm{pH}$ Bereich 6 proteolytische Wirkung der erwähnten Enzyme gespalten, was man als zentrifugale Aufhellung der ursprünglich massiven Trübung des Paracasein-Streifens beobachten kann. In den. Abbildungen erscheinen die Zymogramme als Negative und die Aufhellungen sind dadurch als dunkle Streifen dàrgestellt (Abb. 3).

Aus der Pepsinogen-Fraktionierung der Magenschleimhaut geht die kompliziertere Heterogenität des Fundus gegenüber dem Pylorus hervor. Die Chymosin-Aktivitäten des -Pepsinogens sind deutlich geringer im Verglẹich mit den Aktivitäten des Pepsins, welches vor der eigenen Fraktionierung aus demselben Pepsinogen in einem stark sauren Milieu konvertiert wurde (Abb. 4). Die Chymosin-Aktivität in einer Probe des Magenschleimhaut-Homogenates des Pylorus, welche sich nicht mit der entsprechenden Protease-Aktivität des. Pepsins deckt, konnten wir uns noch nicht erklären (Abb. 5). Das unregelmäßige Vorkommen 'dieses Enzyms, welches als Rennin angesehen werden könnte, fordert noch weitere Beobachtungen.

Im Zymogramm des Duodenalsaftes kann man meist vier Chymosin-Aktivitäten bestimmen, wie aus $\mathrm{Ab}$ bildung 6 ersichtlich ist. Die einzelnen Enzyme haben wir mit den synthetischen Substraten für Chymotrypsin und Trypsin identifiziert. In der Abbildung 7 sind mit der gestrichelten Linie die Gradienten der zwei anodisch und eines schwächeren katodisch lokalisierten Streifens gezeichnet. Das Trypsin kommt als eine homogene
Fundus

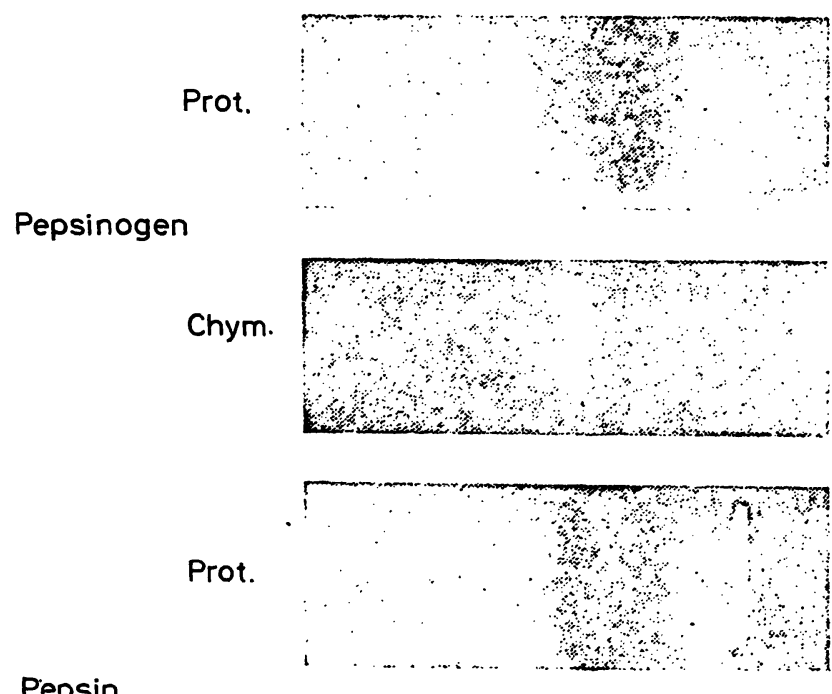

Chym.

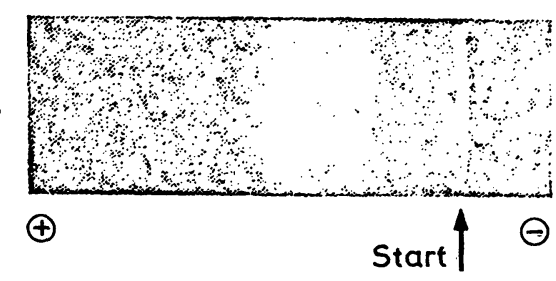

Pylorus
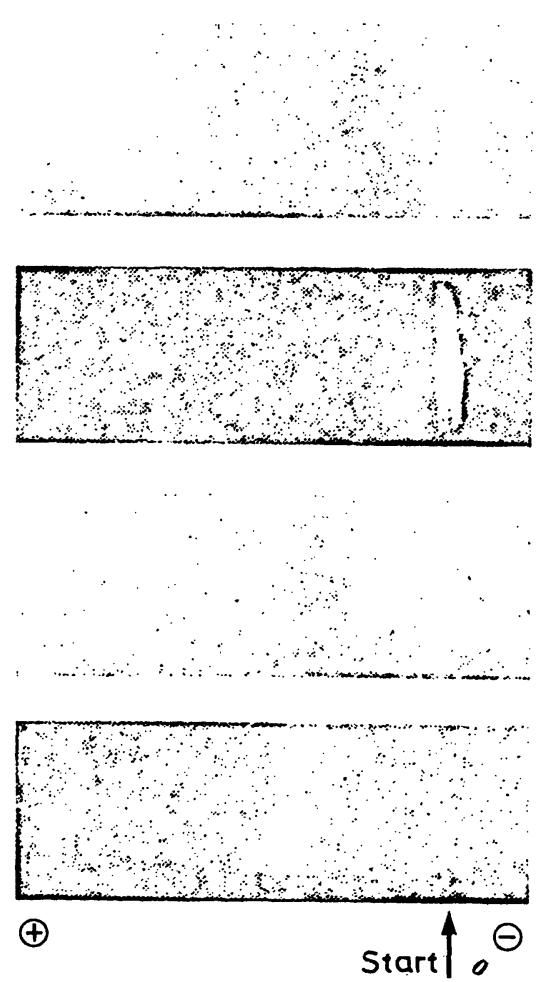

Abb. 4

Die proteolytischen und Chymosin-Aktivitäten der Pepsinogen- und Pepsin-Fraktionen in verschiedenen Magenschleimhaut-Homogenaten Chym. = Chymosin-Aktivität der Pepsinogen- oder Pepsin-Fraktionen. Prot. = proteolytische Aktivitäten; die Detektion der Isoenzyme wurde im stark sauren Milieu durchgeführt 
a

Pylorus

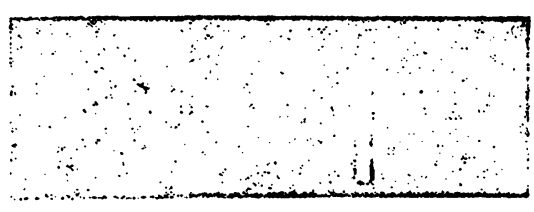

Pepsinogen

Pepsin

$\bigoplus$

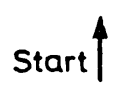

$\Theta$

K.A.

$\oplus$

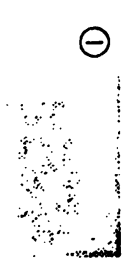

a
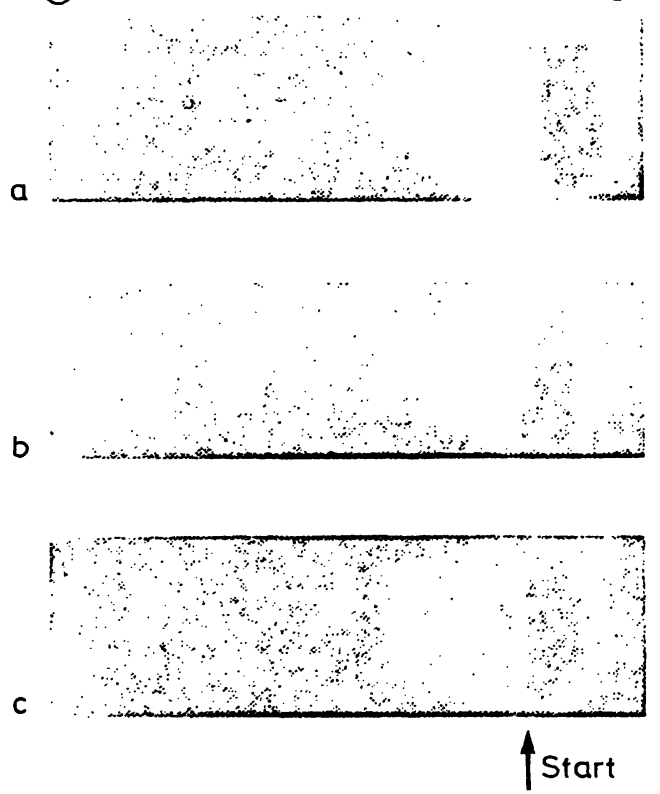

Abb. 6

Die Chymosin-Aktivitäten der Proteasen in drei verschiedenen Proben des Duodenalsaftes, welche von einem Patienten (K. A.) ohne (a) oder nach Stimulation mit $\mathrm{MgSO}_{4}(18 \mathrm{ml}, 250 \mathrm{~g} / \mathrm{l})$ (b) oder mit Vitamin A (c) (10000 I. E.) entnommen wurden

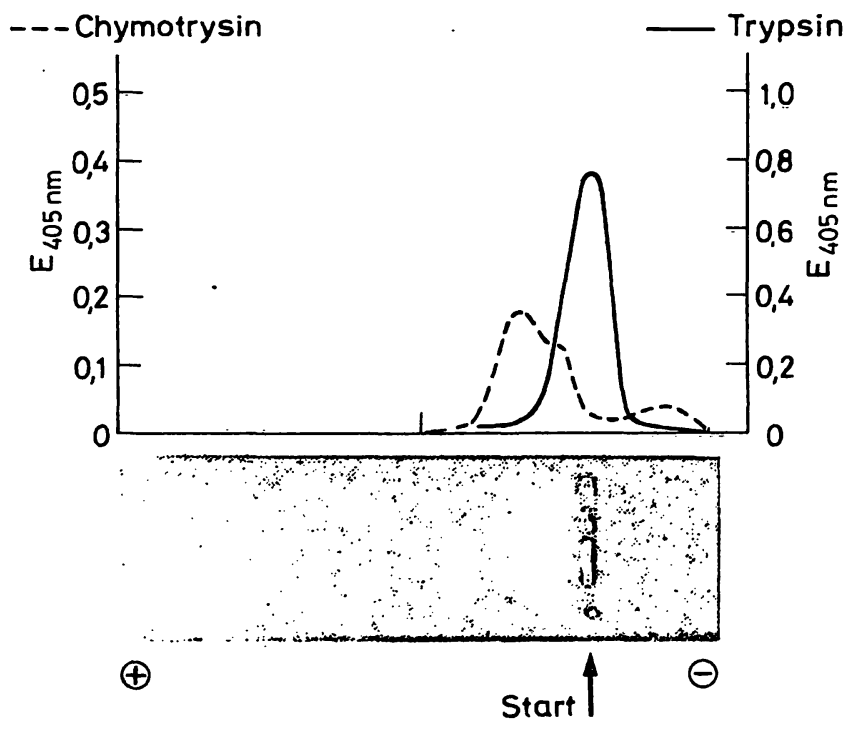

Abb. 7

Die Chymosin-Aktivitäten des Chymotrypsins und Trypsins des Duodenalsaftes. Die Identifizierung dieser Pankreas-Proteasen wurde mit synthetischen Substraten durchgeführt. Siehe Text
syntien

$\oplus$ einzelnen Fraktionen hervor ${ }^{3}$ ). für Pädiatrie, Brno.

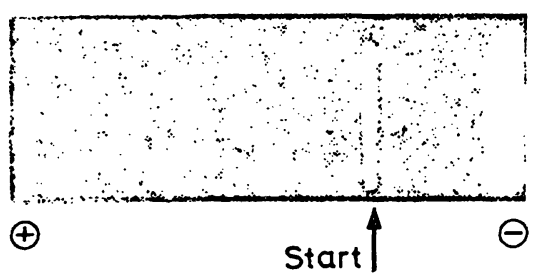

Abb. 5

Atypisch lokalisierte ChymosinFraktion im MagenschleimhautHomogenat des Pylorus nach der elektrophoretischen Trennung $a=$ die proteolytische Aktivität $\mathrm{b}=$ die Chymosin-Aktivität

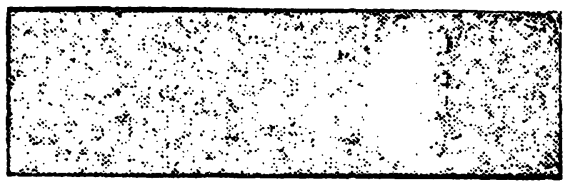

Ja.W.

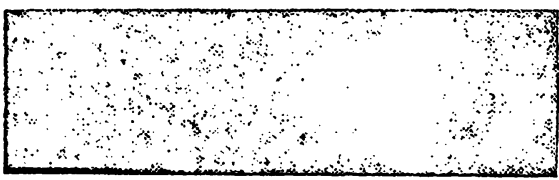

$\Theta$

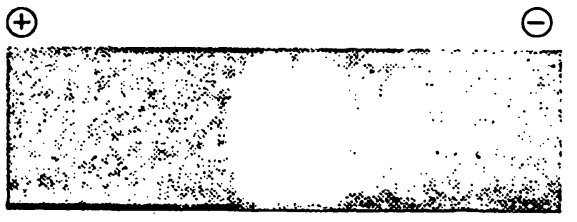

R.G.

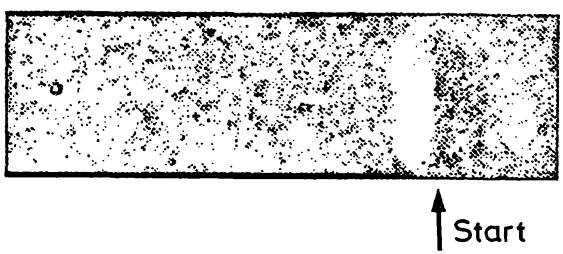

S.G.

Abb. 8

Die Chyınosin-Aktivitäten der Proteasen des Duơdenalsaftes von zwei Zwillingspaaren

Fraktion in der Region des Startes vor und wird dadurch charakterisiert, daß bei der Detektion mit dem Substrat Casein in der Regel unmittelbar nach der Umwandlung in Paracasein dieses gespalten wird, was sich als Aufhellung der Trübung zeigt.

Abbildung 8 zeigt die Zymogramme von zwei zweieiigen Zwillingspaaren, auf welchen auf Grund der Chymosin-Aktivität die Lokalisation der Pankreas- und der Magen-Proteasen ersichtlich ist. Aus den Untersuchungen der Chymosin-Aktivitäten der DuodenalsaftProteasen gehen die individuellen Unterschiede in der Lage und Form sowie auch in den Aktivitäten der

3) Für die Eiigkeitsdiagnose danken wir Frau Dr. med. A. Kubíkoví, C. Sc., Abteilung der Physiologie des Forschungsinstitutes 
In Übereinstimmung mit den Befunden von CoAN et al. (3) zeigen die Zymogramme drei Chymotrypsin-Fraktionen, die alle Chymosin-Aktivität aufweisen. Das Trypsin zeigt sich als einzelne homogene Fraktion in der Nähe des Startes mit maximaler Chymosin-Aktivität. Wenn auch dieser Beitrag vielmehr den Charakter eines Vergleichs bietet, geht aus den Ergebnissen hervor, daß die wichtigsten Magen- und Pankreas-Proteasen aufgrund der Chymosin-Wirkung mit einem einzigen Substrat und bei gleichen $\mathrm{pH}$ Bedingungen nachgewiesen werden können.

Für die technische Mitarbeit danken wir Frau H. MašAráková und Frau D. MATuLoví.

\section{Literatur}

1. SAMLOFF, I. M. (1971), Gastroenterology 60, 586-604. 2. CoAn, M. H., Roberts, R. C. \& Travis, J. (1971), Biochemistry 10, 2711-2716. - 3. COAN, M. H. \& Travis, J. (1972), Biochim. Biophys. Acta 268, 207-211. - 4. Robinson, L. A., Kim Won J., WhITE, T. T. \& HADORN, B. (1972), Scand. J. Gastroentol. 7,
43-45. - 5. KAMARÝ, J. \& Mechloví, V. (1970), diese Z., 8, 567-569. - 6. SEIJfFers, M. J. \& TKatch, R. (1970), Gastroenterology 59, 528-533. - 7. Seijffers, M. J., MrLler, L. L. \& Segal, H. L. (1964), Biochemistry 3, 1203-1209.

RNDr. Jaromir Kamarýt, C. Sc. Forschungsinstitut für Pädiatrie Cernopolni 9 , 66262 Brno; ČssR 